\title{
The role of home healthcare in managing cancer-related pain during COVID-19 pandemic: 'The Triple Triage Protocol'
}

\author{
Tolga Ergönenç ${ }^{1,2,3}$, Jalan Şerbetçigil Ergönenç ${ }^{4}$, Ece Yamak Altinpulluk ${ }^{1,5,6,7}$ \\ 1. Madrid Morphological Research Center (MoMaRC), Ultradissection Spain EchoTraining School, Madrid, Spain. \\ 2. The Republic of Turkey Ministry of Health Akyazı Pain and Palliative Care Center, Sakarya, Turkey. \\ 3. Anesthesia and Reanimation, Sakarya Education and Research Hospital, Sakarya, Turkey. \\ 4. Department of Home Healthcare, Sakarya Education and Research Hospital, Sakarya, Turkey. \\ 5. Department of Outcomes Research Anesthesiology Institute Cleveland Clinic, Cleveland, OH, USA. \\ 6. Department of anesthesiology and Reanimation, Istanbul University-Cerrahpasa, Cerrahpasa Medical Faculty, Istanbul, \\ Turkey. \\ 7. Anesthesiology Clinical Research Office, Ataturk University, Erzurum, Turkey.
}

Correspondence: Tolga Ergonenc MD, E-mail: tolgaergonenc@sakarya.edu.tr, ORCID: https://orcid.org/0000-0002-4325-695X

\section{Abstract}

The coronavirus disease 2019 (COVID-19) can be responsible for severe acute respiratory syndrome and death. To limit the infection spread, non-urgent surgical procedures, day procedures, including interventional pain management, and patient visits, have been postponed or interrupted during the COVID-19 pandemic. Pain management is defined as a fundamental human right, but the rapidly changing nature of the COVID-19 outbreak requires revisions in clinical practice for chronic pain. This article describes the role of home healthcare services in managing cancer pain based on clinical practice during the COVID-19 pandemic and emphasizes the triple triage concept and the use of telemedicine.

Key words: Coronavirus; COVID-19; Telemedicine; Home care; Cancer Pain

Citation: Ergönenç T, Ergönenç JS, Altınpulluk EY. The role of home healthcare in managing cancer-related pain during COVID-19 pandemic: 'The Triple Triage Protocol'. Snaesth. Pain intensive care 2021;25(1):1-4.

DOI 10.35975/apic.v25i1.1430

Received: 4 January 2021, Reviewed \& Accepted: 8 January 2021

\section{Introduction}

The International Association for the Study of Pain (IASP) defines chronic pain as persistent or recurrent pain lasting more than three months after the normal tissue healing. ${ }^{1}$ Chronic pain causes the limitation of daily activities and reduced quality of life. The prevalence of chronic pain in the general population is $\% 20{ }^{2,3}$ Pain is a frequent and devastating symptom in patients with cancer. Moderate to severe pain has been reported at $38.0 \%$ among patients with cancer, resulting in impaired functional status and reduced quality of life. ${ }^{4}$

Severe acute respiratory syndrome coronavirus 2 (SARS-CoV-2) causes the new COVID-19 infection responsible for severe acute respiratory syndrome and death. At the time of writing, 83.3 million patients have been infected by COVID-19, and 1.8 million people at least have died. The COVID-19 pandemic has pushed healthcare systems worldwide to review the deployment of resources. Due to the challenge of controlling the infection, healthcare professionals focused on intensive care and other COVID-19 
dedicated units. While healthcare workers, even in the middle of the COVID-19 duties, chronic pain patients significantly affected cancer pain, still need support.

This article presents home healthcare services' role in managing cancer pain based on clinical practices during the COVID-19 pandemic. It includes arranging home healthcare units to recognize, assess, and manage cancer pain and use telemedicine.

\section{Home healthcare during COVID-19 pandemic}

There are two crucial problems in terms of home healthcare in COVID-19. First are the infected asymptomatic patients with COVID-19. The second risk is the healthcare providers who were infected. All healthcare providers must pay attention to the standardized processes for infection control, but these processes are not sufficient alone.

\subsection{Triple triage protocol}

The triple triage protocol is used to evaluate the patients. The first triage is a telephonic interview with a call center (444 3833 ) called by patients or relatives from all over the country. This step focuses on patients' requests and demographic data. The second telephonic interview with the patient or relatives is in 15 min after the first step, and it recognizes patients who might have mild forms of COVID-19. It should be remembered that pain is a symptom of COVID-19 infection. The home healthcare nurse asks each patient and relatives who live together if, within the previous $48 \mathrm{~h}$, he or she has experienced muscle or joint pain, loss of smell/taste, fever, breathlessness, or cough. The nurse also asks if he had direct contact with people who had COVID-19. Patients with a positive symptom at the second triage are referred to the 112 Emergency Healthcare Services to follow the national health authority's procedures. The patients who do not meet the possible case definition in terms of COVID-19 and meet the home healthcare service criteria in the second telephonic interview have a patient file opened. The patient file proceeds to the zone-based healthcare unit as a case online for the third interview. The home healthcare nurse schedules a home visit in $24 \mathrm{~h}$, depending on symptom severity, in a third telephonic interview (Figure 1). Symptom severity is assessed with the PERSONS score.

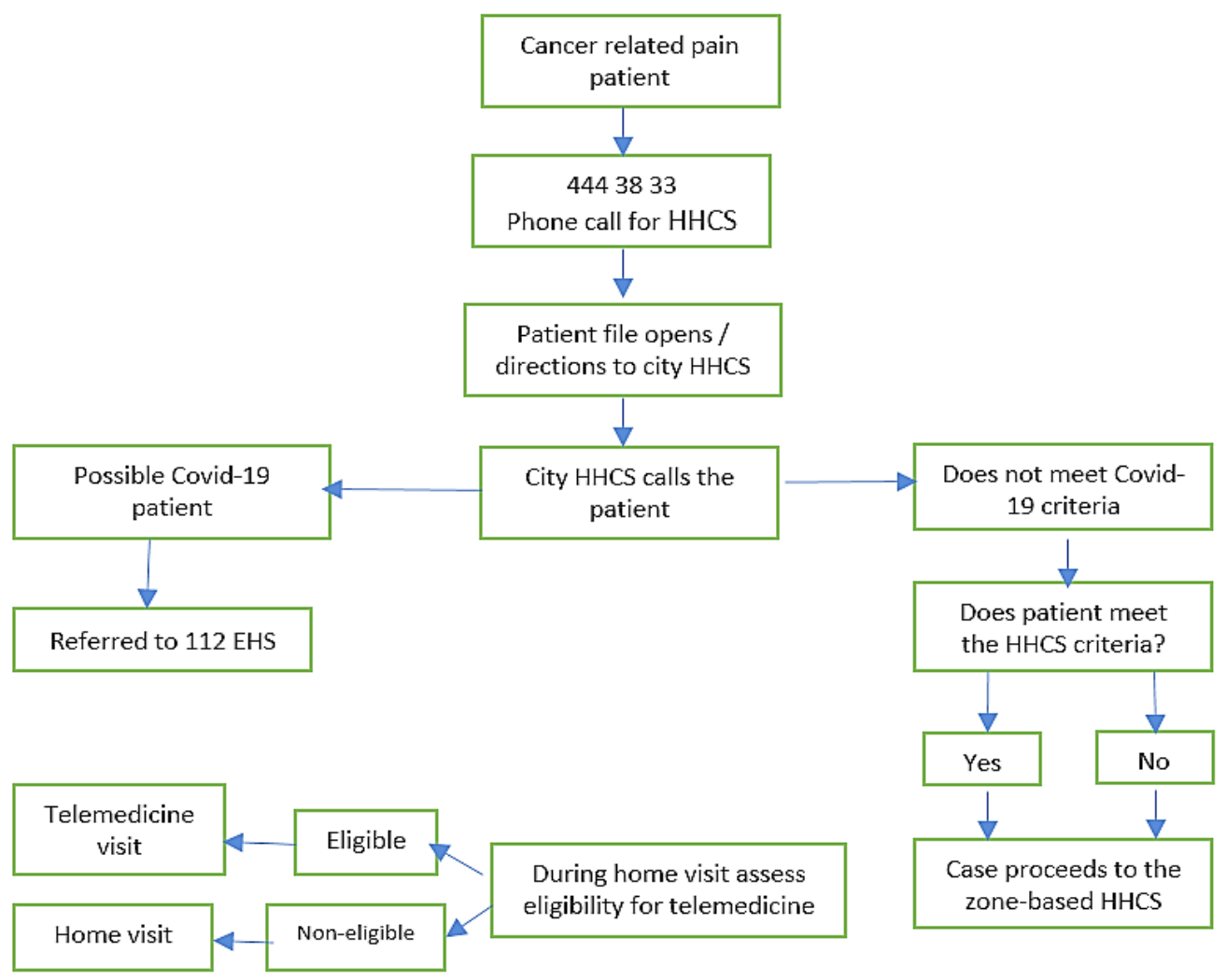

Figure 1: Algorithm of the Home Healthcare Service (HHCS) and Triple Triage System. 
The PERSONS score includes; pain, eating (loss of appetite), rehabilitation (asthenia), sleep (sleep disorders), $\mathrm{O}_{2}$ (dyspnea, cough), nausea/emesis, and suffering (anxiety/depression). ${ }^{5-6}$ Each item is rated on a numeric scale between 0 (no burden) and 10 (worst imaginable burden). Thanks to this process, healthcare professionals do not make unnecessary visits to care for patients and protect patients and themselves from infection risk. Besides, the triple triage system not only offers an effective way to ensure the continuity of the home healthcare system but also to patients with healthcare needs are reached.

\subsection{Continuing cancer pain management}

The patients diagnosed with cancer may experience an amount of pain due to tumor behavior or cancer treatment. Cancer-related pain treatment is an ethical responsibility of healthcare professionals. All treatments needed for cancer pain relief should be continued to relieve suffering, even in pandemic conditions. $^{7}$

When considered urgent procedures for cancer-related pain, the home healthcare team schedules an appointment with the pain clinic during the home visit. European Society of Regional Anesthesia and Pain Therapy (ESRA) and The American Society of Regional Anesthesia and Pain Medicine (ASRA) were announced urgent procedures for cancer-related pain during the pandemic are intrathecal pump malfunction, infection, and refilling. ${ }^{8}$ Intractable cancer pain is semi-urgent. ${ }^{8}$ Cancer patients' clinical conditions are common at risk of progression, so sometimes interventional procedures may offer the best option to improve their quality of life in semi-urgent conditions. $^{9,10}$ Although interventional procedures reduce opioid consumption ${ }^{11}$ and improve the quality of life, these procedures for patients with cancer usually need hospitalization for a couple of days. Patients with cancer have defective immune responses owing to different reasons, and they are more sensitive to infections than the general population. These patients are commonly older and have more comorbidities. $^{12}$ Intensive care admission and respiratory distress or death ${ }^{13}$ are more than the normal population. Potential benefits and possible risks should be considered due to hospitalization can increase patients' exposure to infection. The best choice is to decide the case by case.

Close cooperation between the home healthcare team and the hospital team that undertakes the patient with cancer's pain and palliation management is also required for other than emergency and semiemergency situations. The team that visits the patient at home and evaluates them in all aspects is the home healthcare team. The home care team assesses the patient for pain and examines other factors causing pain and focusing on other health problems caused by pain. The team evaluates the cancer patient's quality of life factors such as nutrition, appetite, nausea, vomiting, and sleep, and plans the necessary steps for their development.

During the home visit by the home healthcare team, it is strongly recommended that using personal protective equipment, including an N95 mask, eye protection, face shield, double gloves, and full-body protecting coverage. The patient and relatives should be wearing a surgical mask during the home visit.

\subsection{Telemedicine options in pain management}

Telemedicine defines telecommunication and the electronic exchange of information through audiovisual platforms. Telemedicine has been suggested for treating chronic pain at home in non-emergent conditions as an alternative to clinical evaluation. It is a useful method to ensure the continuity of patient care after discharge from the hospital. While the home healthcare team maintains the continuity of treatment of patients with cancer discharged from palliative care, oncology department, or pain clinic, they use telemedicine besides home visits. Sundararaman et al. reported that in their review of mobile health technology were promising and were likely be widely adopted in the coming years. ${ }^{14}$ Song et al. reported that telemedicine and appropriate triaging with telehealth were useful in managing pain services during COVID19. ${ }^{15}$ At the time of writing this review, telemedicine platforms with advanced security features are established and available by the Ministry of Health. Telemedicine challenges include inadequate access to and unfamiliarity with the technology, especially in the elderly and less educated patients. At this point, the home healthcare team connects patients and hospital consultants via telemedicine equipment. 


\section{Conclusion}

The COVID-19 pandemic is a difficult period which is given stressed medical service for all healthcare workers and patients worldwide. During the COVID19 pandemic, there is a risk of failing to receive treatment for cancer-related pain patients due to many pain clinics are no longer open. During a pandemic crisis, home healthcare units could contribute to patients with cancer-related pain treatment via home visits and telemedicine. Telemedicine gives an excellent opportunity to avoid "missed care" and helping with the triple triage protocol. At present, telemedicine is limited only to follow-up patients with stable pain conditions. Because telemedicine will spread near future, local medical policies may need adapting to the new situation.

\section{Conflict of interest}

None declared by the authors.

\section{References}

1. Classification of chronic pain. Descriptions of chronic pain syndromes and definitions of pain terms. Prepared by the International Association for the Study of Pain, Subcommittee on Taxonomy. Pain Suppl. 1986;3:S1226. [PubMed]

2. Mills SEE, Nicolson KP, Smith BH. Chronic pain: a review of its epidemiology and associated factors in population-based studies. $\mathrm{Br} \mathrm{J}$ Anaesth. 2019 Aug;123(2):e273-e283. [PubMed] DOI: 10.1016/j.bja.2019.03.023

3. Del Giorno R, Frumento P, Varrassi G, Paladini A, Coaccioli S. Assessment of chronic pain and access to pain therapy: a cross-sectional population-based study. J Pain Res. 2017;10:2577-2584. [PubMed] DOI: 10.2147/JPR.S136292

4. Van den Beuken-van Everdingen $M H$, Hochstenbach LM, Joosten EA, Tjan-Heijnen VC, Janssen DJ. Update on prevalence of pain in patients with cancer: systematic review and meta-analysis. J Pain Symptom Manage. 2016;51(6):1070-1090.e9. [PubMed] DOI: 10.1016/j.jpainsymman.2015.12.340

5. Cortellini A, Porzio G, Masel EK, Berghoff AS, Knotzer $B$, Parisi $A$, et al. The PERSONS score for symptoms assessment in simultaneous care setting: A pilot study. Palliat Support Care. 2019 Feb;17(1):82-86. [PubMed] DOI: $10.1017 /$ S1478951518000238
6. Cortellini A, Porzio G, Cofini V, Necozione S, Parisi A, Peris $F$, et al. The PERSONS score: A new tool for cancer patients' symptom assessment in simultaneous care and home care settings. Palliat Support Care. 2020 Feb;18(1):33-38. [PubMed] DOI: $\underline{10.1017 / S 1478951519000543}$

7. Weinstein SM, Janjan N. Management of Pain. 2015 Jun 1. Available from: https://www.cancernetwork.com/view/managementpain. (Accessed on 03 Jan 2022)

8. American Society of Regional Anesthesia, Pain Medicine. Recommendations on Chronic Pain Practice during the COVID-19 Pandemic. A Joint Statement by American Society of Regional Anesthesia and Pain Medicine (ASRA) and European Society of Regional Anesthesia and Pain Therapy (ESRA). Available from: https://www.asra.com/page/2903/recommendations-onchronic-pain-practice-during-the-covid-19- pandemic. (Accessed on 03 Jan 2022)

9. Shanthanna $H$, Strand $\mathrm{NH}$, Provenzano DA, Lobo CA, Eldabe S, Bhatia A, et al. Caring for patients with pain during the COVID-19 pandemic: consensus recommendations from an international expert panel. Anaesthesia. 2020;75(7):935-944. [PubMed] DOI: 10.1111/anae.15076

10. Cohen SP, Baber ZB, Buvanendran A, McLean BC, Chen $Y$, Hooten WM, et al. Pain management best practices from multispecialty organizations during the COVID-19 pandemic and public health crises. Pain Med. 2020;21(7):1331-1346. [PubMed] DOI: 10.1093/pm/pnaa127

11. Thapa D, Rastogi V, Ahuja V. Cancer pain managementcurrent status. J Anaesthesiol Clin Pharmacol. 2011;27(2):162-168. [PubMed] DOI: 10.4103/0970$\underline{9185.81820}$

12. Extermann M. Measuring comorbidity in older cancer patients. Eur J Cancer. 2000;36(4):453-471. [PubMed] DOI: $10.1016 /$ s0959-8049(99)00319-6

13. Liang W, Guan W, Chen R, Wang W, Li J, Xu Ke, et al. Cancer patients in SARS-CoV-2 infection: a nationwide analysis in China. Lancet Oncol. 2020;21(3):335-337. [PubMed] DOI: 10.1016/S1470-2045(20)30096-6

14. Sundararaman LV, Edwards RR, Ross EL, Jamison RN. Integration of mobile health technology in the treatment of chronic pain: A critical review. Reg Anesth Pain Med. 2017;42(4):488-498. [PubMed] DOI: 10.1097/AAP.0000000000000621

15. Song XJ, Xiong DL, Wang ZY, Yang D, Zhou L, Li RC. Pain management during the COVID-19 pandemic in China: lessons learned. Pain Med. 2020 Nov 7;21(7):1319-1323. [PubMed] DOI: $10.1093 / \mathrm{pm} /$ pnaa143 